\title{
Charged Higgs in Beyond the Minimal Supersymmetric Standard Model at the LHC
}

\author{
Katri Huitu ${ }^{* \dagger}$ \\ Department of Physics, University of Helsinki \\ E-mail: katri.huitu@helsinki.fi
}

\begin{abstract}
The charged Higgs boson is an inevitable particle in supersymmetric models, both in the minimal version and in extensions. It is also a particle, which may have different decay channels depending on the scalar representations in the model, and thus it may help in identifying the model. In this talk I will consider the simplest singlet and triplet extensions of the minimal supersymmetric standard model, and in particular, describe some smoking gun signals of charged Higgs at the LHC collider. I will also comment the charged scalars in a supersymmetric left-right model.
\end{abstract}

Prospects for Charged Higgs Discovery at Colliders

3-6 October 2016

Uppsala, Sweden

\footnotetext{
* Speaker.

${ }^{\dagger}$ The research presented here has been done in collaborations with P. Bandyopadhyay, S. DiChiara, M. Frank, D.K. Ghosh, A.S. Keceli, S. Niyogi, S.K. Rai, I. Saha, H. Waltari [1, 2, 3, 4].
} 


\section{Introduction}

A scalar particle, the Higgs boson, found at the LHC in 2012 opened a new era in particle physics. Since then the literature on the Higgs sector and on its extensions has been growing quickly. It is widely thought that the scalar sector may provide hints going beyond the Standard Model, since many of the burning problems in the Standard Model (SM) may be related to the scalar sector: possibly (pseudo)scalar dark matter, generation of neutrino masses using new scalars, generation of fermion mass hierarchies, etc. Supersymmetry is a general framework, where it is natural to have scalars in addition to fermions and gauge bosons in the model describing Nature.

In supersymmetric extensions of the SM, an additional Higgs doublet is needed in order to cancel the anomalies and to generate masses both to up- and down-type quarks. The SM gauge bosons need three scalar degrees of freedom for their longitudinal component, thus leaving five (pseudo)scalar components, which will form five physical Higgs bosons, two neutral scalars $(h, H)$, one neutral pseudoscalar $(a)$ and a charged Higgs boson $h^{ \pm}$. Thus any supersymmetric extension of the Standard Model necessarily includes at least one charged Higgs boson. On the contrary, neutral (pseudo)scalars could result from singlet nonsupersymmetric extensions of the Standard Model.

In this talk, I discuss the charged Higgs in simple extensions of the minimal supersymmetric standard model (MSSM). After reviewing the relevant features in the MSSM in view of the mentioned extensions, I discuss the model, in which one singlet is added to the MSSM (NMSSM) [5]. The simplest extension adding a triplet field is the triplet extended supersymmetric model (TESSM), which contains one hypercharge zero triplet in addition to the MSSM representations [6]. These simple models show some essential new features in charged Higgs phenomenology, when new representations are added. I also shortly discuss novelties coming up when the gauge sector is extended in the case of a supersymmetric left-right model (SUSYLR) .

In particular the production and decay modes depend on the particular class of models, as well as mass and mass relations. For the purpose of identifying the model such features are important.

\section{Charged Higgs in the MSSM}

The prototype of the charged Higgs in supersymmetric models is the MSSM. The charged Higgs in two Higgs doublet model type-2, which also MSSM is, is well studied, see e.g. [7]. In the MSSM the tree-level masses can be easily found from the $2 \times 2$-matrices, when MSSM is conserved. It is found that if the lightest Higgs is clearly lighter than the others, then the remaining heavy scalar, pseudoscalar and the charged Higgs are nearly degenerate [8].

The decay modes of the charged Higgs can be found and it turns out that irrespective of the ratio of VEVs $(\tan \beta)$, the charged Higgs decays to $\tau \nu_{\tau}$ until it is kinematically possible to decay to top and bottom. Similarly the favored production channels are found to be through gg fusion and through gb fusion.

The charged Higgs has been searched for at the LHC. The charged Higgs lighter than the top quark was looked for at the Run 1. At Run 2 also heavier than top-quark charged Higgs has been serached for in the fermionic decay modes. In the case of Georgi-Machacek -model also $H^{+} \rightarrow W^{+} Z$ was considered. No hints of the charged Higgs has been found, and the mass limit 
from the studied decay modes have been set to $1-2 \mathrm{TeV}$. If the branching ratios to other channels are significant, the mass limit can be reduced accordingly.

\section{Extensions of MSSM}

The scalar sector can contain other representations in addition to the two doublets considered in the MSSM. A severe constraint on models with additional representations comes from the electroweak $\rho$-parameter, which can be written at the tree-level as [8]

$$
\rho=\frac{m_{W}^{2}}{m_{Z}^{2} \cos ^{2} \theta_{W}}=\frac{\Sigma_{T, Y}\left(4 T(T+1)-Y^{2}\right)\left|v_{T, Y}\right|^{2} c_{T, Y}}{\Sigma_{T, Y} 2^{2}\left|v_{T, Y}\right|^{2}},
$$

where $T$ is the weak isospin, $Y$ is hypercharge, $v_{T, Y}$ is the vacuum expectation value (VEV), and $c_{T, Y}$ is $1(0)$ for complex (real) representation. Experimentally $\rho=1.0004_{-0.0004}^{+0.0003}$. For singlets and doublets $\rho=1$ at the tree level, but it constrains models with triplets, if the contributions of various particles do not cancel. Strict cancellation of the triplet VEVs is found in the Georgi-Machacek -model.

\subsection{The singlet extension, NMSSM}

The $\mu$-parameter of the MSSM could be generated without a new scale in the superpotential if a new singlet is added, as happens NMSSM [5]. In NMSSM the superpotential is

$$
W_{N M S S M}=W_{M S S M}^{\mu=0}+\lambda_{S} S H_{u} \cdot H_{d}+\frac{1}{3} \kappa S^{3},
$$

where the effective $\mu$-term is $\lambda\langle S\rangle$. There are two new degrees of freedom in the scalar sector of the model, and thus the particle content is three scalars, $h_{i}, i=1,2,3$, two pseudoscalars, $a_{1}, a_{2}$ and the charged scalars $h^{ \pm}$. In NMSSM there are new contributions to the lightest Higgs mass alleviating fine tuning of Higgs mass,

$$
\Delta m_{h}^{2}=\frac{\lambda^{2}}{g^{2}} M_{Z}^{2} \sin ^{2} 2 \beta
$$

The charged Higgs remains in doublet, and thus it couples similarly to the fermions than the charged Higgs in the MSSM. The lightest particle in NMSSM Higgs sector can be the lightest pseudoscalar. Such a pseudoscalar has been searched for at the LHC from $h_{1} \rightarrow a a$. This search has not been successful, and we will assume here that $m_{a}>m_{h_{1}} / 2$. The search of the charged Higgs in top decays has been equally unsuccessful, and consequently we assume that a charged Higgs is heavier than the top quark. There is still a possibility that the charged Higgs is only slightly heavier that the top quark, and that the pseudoscalar could be slightly too heavy for the on-shell decay of the Higgs boson. Then one could entertain a possibility of the charged Higgs decays to the pseudoscalar and a $\mathrm{W}$ boson [1], which is a distinctly different signal compared to the MSSM charged Higgs decay signals.

Since the charged Higgs couplings to the SM particles is the same than in the MSSM, the production of the charged Higgs in NMSSM is similar than in the MSSM. However, because of the new particles in the Higgs sector, the decay of the charged Higgs is different. It is possible to find benchmark points where

$$
h^{ \pm} \rightarrow a W^{ \pm}
$$


is large or even the dominant decay mode (in our benchmark points the branching ratio varies between $18 \%$ and $66 \%$ ).

We have studied the possibilities for detection in three benchmark points, where the main production channel is $b g \rightarrow t h^{ \pm}$, and the pseudoscalar from the charged Higgs decay decays to taus ( $\mathrm{BR} \sim 10 \%$ ) or to the b-quarks ( $\mathrm{BR} \sim 90 \%$ ), and the two $\mathrm{W}$ in the process decay either leptonically or hadronically. We found that, if $a \rightarrow \tau \tau$, the increased statistics when one $\mathrm{W}$ decays hadronically is useful in increasing the significance of the signal compared to the background, and for some benchmark points the signal could be seen in early LHC run-2. On the other hand, search with $a \rightarrow b b$ does not seem successful because of large background even if both Ws decay leptonically.

\subsection{The triplet extension, TESSM}

In addition to singlet or doublet extensions, several triplet extensions exist. The simplest possible supersymmetric triplet model can be constructed with a hypercharge zero triplet [6]. Such a representation does not lead to new contributions for the triangle anomaly, and thus it is enough to add a single triplet to the MSSM superpotential:

$$
W_{T E S S M}=W_{M S S M}^{\mu=0}+\lambda H_{d} \cdot T H_{u}+\mu_{D} H_{d} \cdot H_{u}+\mu_{T} \operatorname{Tr}(T T),
$$

where $T$ is a triplet superfield,

$$
T=\left(\begin{array}{cc}
\sqrt{\frac{1}{2}} T^{0} & T_{2}^{+} \\
T_{1}^{-} & -\sqrt{\frac{1}{2}} T^{0}
\end{array}\right),
$$

$\mu_{D}$ is the Higgs doublet mixing parameter and $\mu_{T}$ the Higgs triplet mass parameter. In the triplet there is one new neutral scalar and two new charged scalars. Thus the number of neutral scalars and pseudoscalars is the same than in the NMSSM, while the number of charged scalars triples.

As discussed, the triplet representation restricts the possible VEV of the neutral component due to contribution to the gauge boson masses, and it is found that $\left\langle T^{0}\right\rangle<5 \mathrm{GeV}$. This limit makes it impossible to make the model scale invariant in the same way than the NMSSM, since without $\mu_{D}$ such a small value of the triplet VEV produces a light chargino, excluded by experiments. The experimentally observed Higgs boson mass has new tree-level contributions [9] which alleviates the fine tuning [4]:

$$
\Delta m_{h}^{2}=\frac{2 \lambda^{2}}{g_{2}^{2}+g_{1}^{2}} M_{Z}^{2} \sin ^{2} 2 \beta .
$$

Also the radiative contributions from electroweak sector may be quite large [10]. The new contributions are bigger for smaller $\tan \beta$ and thus our benchmarks have $\tan \beta \sim 5$. One may worry about the rare decay $b \rightarrow s \gamma$ for these benchmarks, since there are several new charged Higgses and charginos, contributing to the decay. It turns out that the lightest charged Higgs for our benchmarks is dominantly triplet, and since it does not couple directly to fermions, the contribution to such rare decays is tiny [4].

The qualitatively new coupling in the case of triplets compared to doublets or singlets is treelevel $H^{ \pm} Z W^{\mp}$, which in MSSM is only radiatively generated [11, 12]. In our benchmarks the dominantly triplet charged Higgs has both large contributions to the production through this coupling, as well as the decay of the charged Higgs can have a major branching ratio to $\mathrm{Z}$ and $\mathrm{W}$, see Fig. 1. 


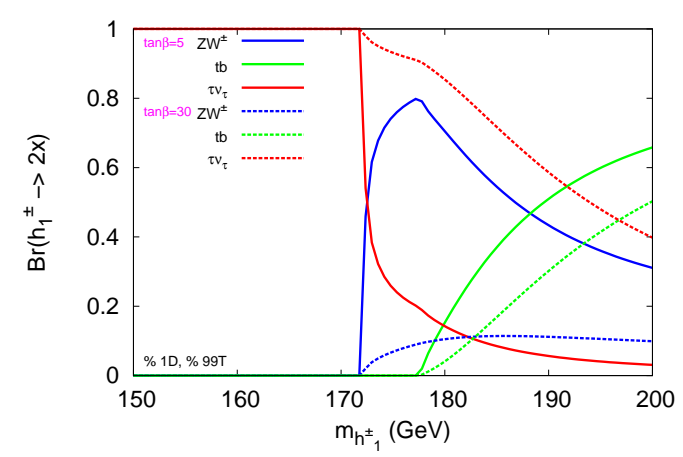

Figure 1: The branching ratios of the lightest charged Higgs for a benchmark scenario [4].

We analyzed in [2] several benchmark points with large triplet contribution in multilepton final states. It seems that three lepton final states can be detected in favourable part of the parameter space, while four to six lepton final states require more than $1000 \mathrm{fb}^{-1}$. Interestingly, since the lightest charged Higgs in TESSM needs not be degenerate with the lightest pseudoscalar or second lightest neutral Higgs - contrary to the MSSM - one can get charged Higgs through $a_{1}$ or $h_{2} \rightarrow$ $h_{1}^{\mp} W^{ \pm}$. This makes it possible to see kinematical edges in the kinematical distributions, from which information also on these heavier Higgs bosons may be deduced [2].

\subsection{Some comments on supersymmetric left-right model}

A particularly well-motivated model with triplets is the supersymmetric left-right model based on the gauge group $S U(3)_{c} \times S U(2)_{R} \times S U(2)_{L} \times U(1)_{B-L}$, in which the triplets may produce neutrino masses via the type-II seesaw mechanism. Because of the left-right symmetry, the righthanded fermions exist in $S U(2)_{L}$ doublets, making the right-handed neutrino a necessary ingredient in the model. The $S U(2)_{R} \times U(1)_{B-L}$ symmetry can be broken by a right-handed triplet. The corresponding VEV needs to be at least at a few $\mathrm{TeV}$ scale, in order to satisfy the experimental limits for the new heavy gauge bosons. There is no need for the $S U(2)_{L}$ triplet VEV, even if they are included in the model because of the left-right-symmetry. This makes the model natural in view of the $\rho$-parameter.

The Higgs sector of the supersymmetric left-right model becomes quite extensive because of the anomaly cancellation requirement. Most of the new physical scalars, however, can be assumed heavy, partly because of the large right-handed triplet VEV [3].

The lightest neutral, pseudoscalar and charged Higgs bosons of the SUSYLR model in our benchmark points are mostly doublets and close to each other in mass [3]. The lightest doubly charged Higgs boson is always light [13]. In the model described in [3] most of the doubly charged scalar mass comes from slepton-lepton radiative corrections leading to a relatively large coupling of tau-leptons to the Higgs and to a large branching ratio of the doubly charged Higgs to a pair of same-sign tau-leptons. This may be the first signal of the model. Other interesting signals can come from the right-handed gauge boson leptonic decays, if $W_{R}$ is not too heavy [14]. 


\section{Summary}

The singlet and triplet extensions of MSSM can lead to alleviated fine tuning of the Higgs mass. In the discussed extensions interesting new signals for the charged Higgs can be found. The discussed charged Higgs decay to a pseudoscalar and $W$ cannot happen in MSSM. Similarly processes originating from tree-level $H^{ \pm} W^{\mp} Z$ are not present in MSSM or in NMSSM.

Another discussed extension, including extension of the gauge symmetry, is the supersymmetric left-right model, in which many current problems of particle physics can be solved (including neutrino mass generation, parity violation, dark matter, strong and weak $\mathrm{CP}$ violation). The Higgs sector of the model becomes quite large. In addition to the previous signals, the SUSYLR model Higgs sector has new interesting features. E.g. a relatively light doubly charged Higgs which can be easily observable in the searches, if kinematically available.

\section{References}

[1] P. Bandyopadhyay, K. Huitu and S. Niyogi, "Non-standard charged Higgs decay at the LHC in Next-to-Minimal Supersymmetric Standard Model,” JHEP 1607 (2016) 015 [arXiv:1512.09241 [hep-ph]].

[2] P. Bandyopadhyay, K. Huitu and A. Sabanci Keceli, "Multi-Lepton Signatures of the Triplet Like Charged Higgs at the LHC," JHEP 1505 (2015) 026 [arXiv:1412.7359 [hep-ph]].

[3] M. Frank, D. K. Ghosh, K. Huitu, S. K. Rai, I. Saha and H. Waltari, "Left-right supersymmetry after the Higgs boson discovery," Phys. Rev. D 90 (2014) no.11, 115021 [arXiv:1408.2423 [hep-ph]].

[4] P. Bandyopadhyay, S. Di Chiara, K. Huitu and A. S. Keceli, "Naturality vs perturbativity, B physics, and LHC data in triplet extension of MSSM," JHEP 1411 (2014) 062 [arXiv:1407.4836 [hep-ph]].

[5] U. Ellwanger, C. Hugonie and A. M. Teixeira, "The Next-to-Minimal Supersymmetric Standard Model," Phys. Rept. 496 (2010) 1 [arXiv:0910.1785 [hep-ph]].

[6] J. R. Espinosa and M. Quiros, "Higgs triplets in the supersymmetric standard model," Nucl. Phys. B 384 (1992) 113.

[7] A. G. Akeroyd et al., "Prospects for charged Higgs searches at the LHC," arXiv:1607.01320 [hep-ph].

[8] J. F. Gunion, H. E. Haber, G. L. Kane and S. Dawson, “The Higgs Hunter’s Guide,” Front. Phys. 80 (2000) 1 .

[9] S. Di Chiara and K. Hsieh, "Triplet Extended Supersymmetric Standard Model," Phys. Rev. D 78 (2008) 055016 [arXiv:0805.2623 [hep-ph]].

[10] P. Bandyopadhyay, K. Huitu and A. Sabanci, "Status of $Y=0$ Triplet Higgs with supersymmetry in the light of $125 \mathrm{GeV}$ Higgs discovery," JHEP 1310 (2013) 091 [arXiv:1306.4530 [hep-ph]].

[11] J. F. Gunion, G. L. Kane and J. Wudka, "Search Techniques for Charged and Neutral Intermediate Mass Higgs Bosons," Nucl. Phys. B 299 (1988) 231.

[12] A. Mendez and A. Pomarol, "One loop induced $H^{+} W^{+} Z$ vertex in the minimal supersymmetry model,” Nucl. Phys. B 349 (1991) 369.

[13] K. Huitu and J. Maalampi, "The Higgs sector of a supersymmetric left-right model," Phys. Lett. B 344 (1995) 217 [hep-ph/9410342].

[14] M. Frank, B. Fuks, K. Huitu, S. K. Rai, and H. Waltari, work in progress. 